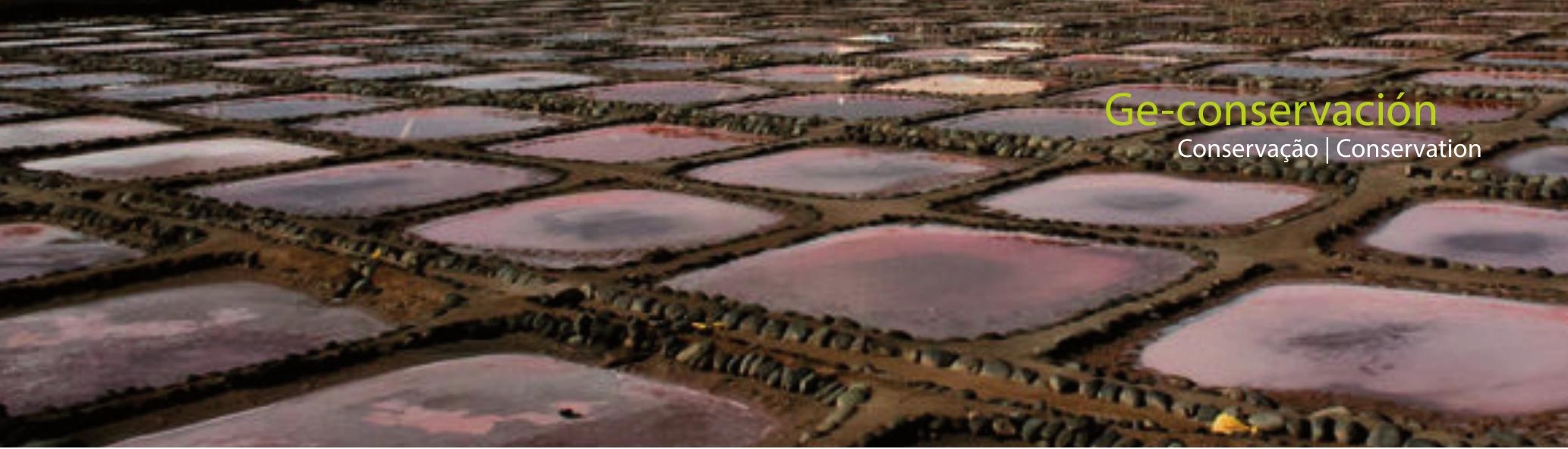

\title{
El paisaje salinero canario. Alternativas para su revitalización
}

\author{
Víctor Manuel Cabrera García
}

\begin{abstract}
Resumen: Las salinas constituyen uno de los paisajes más singulares que la humanidad ha creado al borde del mar y son considerados como espacios etnográficos de gran interés para la ciudadanía. Ante el manifiesto estado ruinoso que presentan las salinas canarias debido al abandono por parte de la sociedad actual de las mismas por falta de actividad, se pretende apostar por la recuperación de las mismas desde un punto de vista paisajístico, creando espacios ajardinados de carácter liquido e iluminados artificialmente en horario nocturno mediante el constaste de las tonalidades de las lámparas. La iniciativa planteada posibilitaría recuperar lo que aún no se ha perdido de estas construcciones tan singulares procedentes del patrimonio industrial tradicional canario y aportarían un valor añadido respecto a las diversas rutas culturales, patrimoniales y paisajísticas pensadas fundamentalmente en horario diurno.
\end{abstract}

Palabras clave: salinas, sal, aeromotores, patrimonio, rehabilitar, conservar

\section{The canary salinary landscape. Alternatives for it revitalization}

Abstract: The saltworks are one of the most unique landscapes that humanity has created on the edge of the sea and are considered ethnographic spaces of great interest to citizens. Given the manifest dilapidated state of the Canary Islands saltworks due to the abandonment by the current society of the same for lack of activity, it is intended to bet on the recovery of them from a landscape point of view, creating landscaped spaces of a liquid nature and artificially illuminated at night by the constrast of the tonalities of the lamps. The proposed initiative would make it possible to recover what has not yet been lost of these singular constructions coming from the traditional Canarian industrial heritage and would provide an added value with respect to the various cultural, heritage and landscape routes thought mainly in daytime schedule.

Keyword: saltworks, salt, windmills, heritage, rehabilitate, conserve

\section{A paisagem salineira das Ilhas Canárias. Alternativas para a sua revitalização}

Resumo: As salinas são uma das paisagens mais singulares que a humanidade criou junto ao mar, consideradas espaços etnográficos de grande interesse para a cidadania. Perante o estado ruinoso das salinas das Ilhas Canárias, devido ao abandono por parte da sociedade atual e por falta de atividade, pretende-se apostar na sua recuperação do ponto de vista paisagístico, criando espaços ajardinados de "carácter líquido", iluminados artificialmente à noite, por meio do contraste de lâmpadas e das suas tonalidades. A iniciativa proposta permitiria recuperar o que ainda não se perdeu dessas construções singulares, provenientes do património industrial tradicional das Canárias e daria um valor adicional às diversas rotas culturais, patrimoniais e paisagísticas, pensadas principalmente para o horário diurno.

Palavras-chave: salinas, sal, aeromotores, património, reabilitar, conservar 


\section{Introducción}

El paisaje salinero es una realidad compleja y dinámica, compuesta por elementos naturales y culturales, materiales e inmateriales, tangibles e intangibles, consecuencia de los procesos de producción y comercialización de la sal sobre el territorio a lo largo de los siglos. Las salinas son, por tanto, paisajes culturales cuya definición viene recogida, entre otros documentos, en el Plan Nacional de Paisaje Cultural como el "resultado de la interacción en el tiempo de las personas y el medio natural, cuya expresión es un territorio percibido y valorado por sus cualidades culturales, producto de un proceso y soporte de la identidad de una comunidad" (Román 2014)

Las salinas son lugares de características únicas resultado de un proceso artesanal de producción y comercialización de la sal, que se obtiene a partir de una serie de construcciones situadas tanto en los territorios costeros como en los de interior. Estos espacios tienen valores naturales, medioambientales, culturales, patrimoniales y sociales resultado de la acción e interacción de factores naturales y humanos en el territorio (Convenio Europeo del Paisaje, 2000)

\section{Las salinas canarias}

Normalmente en las salinas existen dos periodos bien diferenciados, el primero corresponde en el periodo de tiempo del mes de mayo al mes de octubre, conocida como la etapa de zafra en el que las condiciones meteorológicas más favorables permitan la extracción de la sal. El segundo periodo corresponde el intervalo de tiempo entre el mes de noviembre al mes de abril, que es cuando las salinas apenas producen sal y los maestros salineros se limitan a realizar las correspondientes labores de mantenimiento de las instalaciones. Sin embargo, en las salinas canarias apenas existen periodos diferenciados ya que las condiciones climatológicas derivadas de numerosas horas de sol existentes, la presencia constante de los vientos alisios y las escasas precipitaciones favorecen el cultivo de la sal durante todo el año.

El ciclo del cultivo de la sal comienza con la captación del agua del mar a través de los diversos "tomaderos o saltaderos" que puedan existir a nivel del mar y que permiten trasladar el agua del mar hacia los "cocederos o calentadores". Estos elementos se construyen a diferentes niveles ya que se adaptan al terreno donde se construyen y son de dimensiones variables, lo que facilita el trasiego de unos a otros y a su vez permiten el traslado del agua salada hacia otros estanques denominados "tajos o cristalizadores" por gravedad. Cuando los cocederos están a la misma cota que el nivel del mar, la pleamar empuja el mar hacia los tomaderos y mediante una red de canales permite el traslado del agua salada hacia los cocederos. En cambio, cuando los cocederos están a una cota superior respecto a los tomaderos, el llenado de los mismos se realizaba antaño por unos los molinos de viento denominados aeromotores salineros que bombean agua de cotas inferiores a cotas superiores, construidos de torre piramidal de madera y de rotor fundamentalmente de madera y aspas de tela, constituyendo uno de los más antiguos referentes de la explotación de la energía eólica en Canarias. En la actualidad, estas maquinas cólicas lentas están en desuso y están a punto de desaparecer debido a falta de mantenimiento y de actividad. Hoy día para desempeñar esta función se utilizan bombas alimentadas principalmente por combustibles fósiles permitiendo aumentar la productividad en las salinas.

Una vez elevada el agua del mar, la misma es trasladada mediante unos elementos denominados "canales" hacia los cocederos y permanecerá allí expuesta al sol durante un periodo aproximado de nueve días donde se produce un precondensación de la misma elevando su nivel de salinidad. Pasado ese periodo de tiempo, el agua salada se traslada a los tajos, cristalizadores o parcelas de evaporación a través de una red de acequias denominados caños donde permanece durante un periodo de tiempo que oscila entre los tres a ocho días, dependiendo de las condiciones climáticas como lo son la temperatura, la insolación y los vientos. Los maestros salineros participan de esta etapa favoreciendo la ruptura durante al menos dos veces al día de las capas finas de sal que se forman en la superficie de las láminas de agua. Cuando la sal ha alcanzado la densidad adecuada los maestros salineros la suben y la depositan en pequeñas montañas denominadas "balaches" donde permanece allí amontonada aproximadamente durante una semana con la finalidad de que pierda los restos de humedad que pueda poseer, para posteriormente trasladar la sal obtenida hacia el almacén para el empaquetado y su posterior distribución.

Las salinas son espacios a proteger por su alto valor ecológico según la UNESCO, no solo en referencia a la obtención artesanal de la sal, sino que son espacios que por sí mismos poseen una gran riqueza ecológica en cuanto a su flora, fauna y las comunidades que forman. Las especies vegetales más integradas en el ambiente acuático son las diminutas algas microscópicas que viven flotando en el seno del agua y están sujetas a sus movimientos y cambios de composición. Estas algas dependen por completo del agua, constituyen el llamado fitoplacton y son la base para la alimentación de todos los animales que viven en esta comunidad. En las salinas canarias abunda un alga microscópica denominada la Dunaliella salina, siendo esta última la causante de que los tajos adquieran un tono de color rosa. La vida animal en estos ambientes es interesante, pues determinados animales, debido a su movilidad, pueden usar este hábitat sólo temporalmente, de modo que su adaptación es más fácil. Los minúsculos animales que forman el llamado "zooplancton", viven en el seno del agua y están sujetos a sus variaciones en composición y movimientos, teniendo en todo caso, una capacidad de desplazamiento muy 
limitada, ya que son los más dependientes del agua. Es el caso de un pequeño crustáceo que tiene un tamaño que oscila entre los $7 \mathrm{~mm}$ a $15 \mathrm{~mm}$ denominado la Artemia salina, animal filtrador que come pequeñas partículas orgánicas en suspensión y más concretamente, del alga Dunaliella que vive en las salinas canarias.

Las salinas también son espacios de descanso, comida y, a veces, cría para muchas de las aves migratorias acuáticas, que como bien es sabido, realizan desplazamientos periódicos, siguiendo las estaciones del año. Estas aves, bajan hacia el ecuador en busca de lugares más cálidos en los meses de invierno y vuelven en verano a las latitudes más altas, consiguiéndose mantenerse en unos límites de temperatura adecuados para su subsistencia además de alimentarse de las charcas de donde obtienen alimento.

Aunque las referencias salineras más antiguas de las islas Canarias datan del siglo XVI, el primer gran desarrollo de los diversos ingenios salineros en las islas se produce a finales del siglo XVIII, tanto es así se deniegan curiosamente nuevas solicitudes bajo el pretexto de que la creciente producción de sal canaria y su posible destino americano podrían afectar a las extracciones de las sales de La Mata y la Bahía de Cádiz, dos de los baluartes del monopolio real español. A esta época corresponde el gran auge salinero de Gran Canaria que mantuvo durante doscientos años una clara hegemonía en el conjunto insular y que estuvo directamente relacionada con la Pesca de Berbería. La segunda gran eclosión salinera en las islas se produce entre 1910 y 1930, especialmente en Lanzarote y de la mano de una floreciente industria conservera. Sin embargo, el hecho insular condicionó el que dentro de este marco genérico cada isla tuviera una versión propia sobre la historia y la cultura de la sal (Marín; Luengo 1994)

En las Islas Canarias llegaron a existir más de sesenta salinas, en su mayoría localizadas en las islas orientales del archipiélago de las que hoy en día solamente perviven unas pocas, que afortunada y acertadamente se encuentran protegidas, bien sea por su interés arquitectónico siendo declaradas Bien de Interés Cultural (BIC) o por su interés natural como Espacio Natural al ser zonas de gran relevancia para la avifauna, y constituyen claramente uno de los modelos de intervención humana más ejemplar por su calidad de diseño, adaptación al medio, referente de la historia, valor cultural, respeto a la tradición, aportación al medio ambiente y a la riqueza patrimonial de las islas Canarias.

Existen cuatro tipos constructivos de salinas en el archipiélago canario (Marín; Luengo 1994):

- La salina primitiva sobre roca de trazado circular.

- La salina antigua de mortero de cal con tajos de pequeño tamaño.

- La salina de tajo sencillo de barro.

- La salina nueva de tajo de barro con forro de piedra.
Las primeras salinas canarias datan del siglo XVI y se construyeron sobre un lecho de piedra, aprovechando áreas rocosas cercanas a las líneas de marea alta. Las salinas del Bufadero en el municipio de Arucas [figura 1], en la isla de Gran Canaria, son las únicas supervivientes del modelo construidas sobre piedra en todo el Archipiélago, y son las más antiguas de Canarias. Tras una minuciosa restauración, están en pleno funcionamiento. Los "cocederos", que son estanques donde se almacena el agua del mar se localizan próximos a la línea de costa y es aquí empieza el proceso de evaporación del agua salada (sol y viento) que se acelera en otros estanques más pequeños denominados los "tajos" y que se siguen llenando a mano. Es aquí donde el trabajo del maestro salinero es crucial para acelerar el proceso de obtención de la sal mediante el "picado" (ruptura de los cristales de sal de la superficie) y el 'arrollado', es decir, el amontonamiento posterior de la sal en pequeñas montañas para que se favorezca expulsar la humedad de la sal.

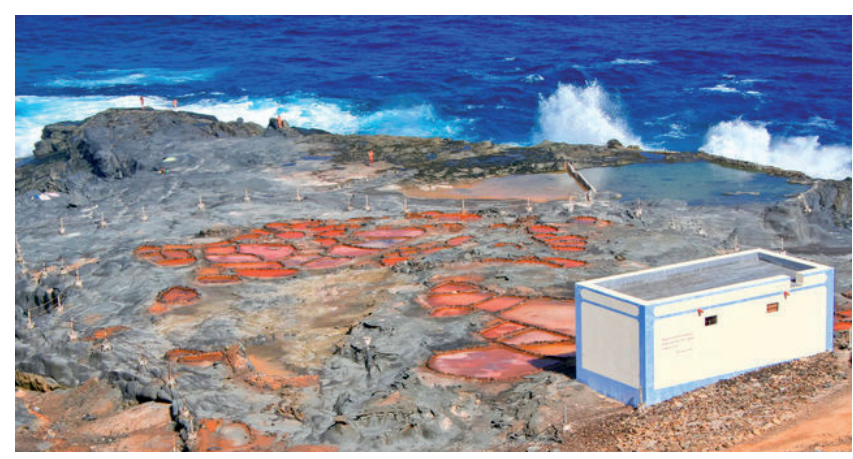

Figura 1.- Salinas del Bufadero, TM de Arucas, Isla de Gran Canaria. Fotografía: https://www.eldiario.es/viajar ahora

Posteriormente, el modelo evolucionó para intensificar la producción de sal al socaire de la expansión de la industria de las salazones derivadas de la pesca. Son las salinas construidas sobre lecho de barro, auténticas infraestructuras hidráulicas con grandes estanques de almacenamiento de agua de mar, molinos de viento para bombear el agua salada de cotas inferiores a cotas superiores respecto al nivel del mar y los complejos sistemas de los cocederos y tajos con una variada red de canalizaciones [figura 2 y figura 3]. Corresponden a este sistema las salinas de Fuencaliente en la isla de La Palma, las salinas de Bocacangrejo, las salinas de la Florida y las salinas de Arinaga en el municipio de Agüimes, las salinas de Tenefé en Pozo Izquierdo en el municipio de Santa Lucía en la isla Gran Canaria, las salinas del Carmen en el municipio de la Antigua en la isla de Fuerteventura, las salinas de los Agujeros en el municipio de Teguise y las salinas de Janubio en el municipio de Yaiza en la isla de Lanzarote.

Las salinas canarias disponen de cinco elementos principales: 
- Sistema de captación e impulsión: Normalmente con la presencia de molinos de viento denominados aeromotores salineros.

- Los caños: Son canalizaciones que permiten la circulación del agua salada hacia los diversos tipos de estanques donde se almacena el agua del mar

- Los cocederos: Son estanques de dimensiones variables donde el agua de mar permanece en un periodo de tiempo que oscila entre cuatro a doce días. - Los tajos: Son estanques más pequeños donde se almacena el agua salada, elevando su salinidad perdiendo que se produzca la cristalización de la sal favorecida por la irradiación solar y la presencia de los vientos alisios.

- El almacén: Se trata de un edificio donde se traslada la sal para su empaquetado y su posterior distribución.

Las salinas son un ingenio industrial de carácter tradicional que poseen múltiples valores añadidos como es la arquitectura, la tecnología y el paisaje de las zonas húmedas. La introducción en las islas Canarias de las técnicas de frío (neveras y congeladores) así como la caída del sector pesquero (salazón) ocasionaron el declive de las salinas y que perdura hasta nuestros días, con lo que actualmente la producción de sal que se obtiene de forma tradicional se centra principalmente en suministrar a la producción interna de las islas como lo son las panaderías, las fabricas de queso, etc. Es notorio que la producción salinera de las islas ya no juega un papel tan importante como lo fue antaño en la economía del archipiélago canario, y que actualmente, corresponde al sector turístico ser el principal sustento económico de las

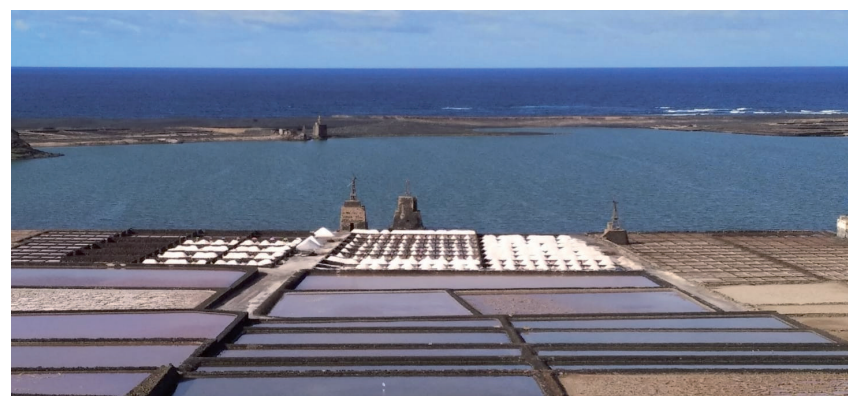

Figura 2.- Salinas de Janubio, TM de Yaiza, Isla de Lanzarote Fotografía: Andrely Peña Roa

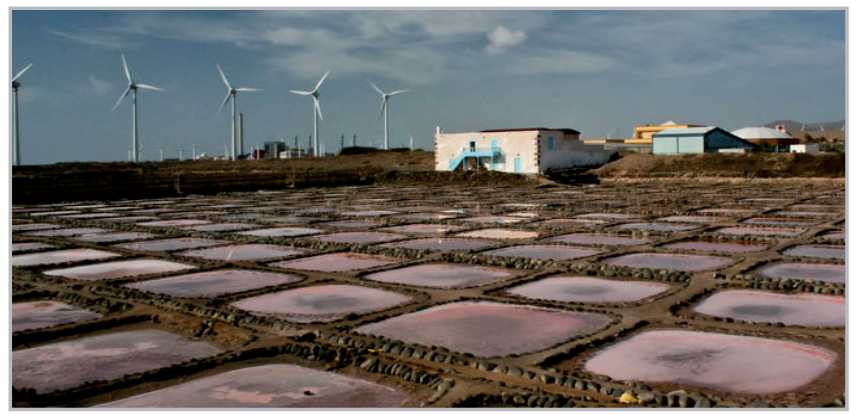

Figura 3.- Salinas de Tenefé, Pozo Izquierdo, Santa Lucía, isla de Gran Canaria. Fotografía: https://www.eldiario.es/viajar ahora islas. No obstante, cabe destacar que desde un punto de vista ambiental estos espacios constituyen un ejemplo de intervención racional en el medio generando un paisaje cultural de gran riqueza estética. Este paisaje cultural está construido por los materiales del entorno lo que favorece la integración con el medio. Por la situación entre la tierra y el mar, las salinas constituyen una marisma artificial que permite la convivencia de un amplio conjunto de animales y de plantas adaptados a estos ecosistemas.

Las emociones que el ser humano siente al contemplar un paisaje están directamente relacionadas con la sensibilidad, la memoria, el aprendizaje y los fenómenos sociales y culturales que lo rodean. De hecho, el paisaje observado por las personas que lo producen, gestionan y viven, en este caso los salineros y vecinos de las salinas, genera emociones y sentimientos distintos que en las personas que lo contemplan por primera vez, como los visitantes o turistas ocasionales. La relación entre el hombre y los paisajes de la sal ha sido representada, a lo largo del tiempo, a través de diversas manifestaciones artísticas (Román 2014).

La presente investigación aborda la situación de abandono y el estado ruinoso que presentan partes concretas de las salinas y muy especialmente, los molinos de viento "aeromotores salineros", debido a que en la actualidad no se utilizan como elementos indispensables para el bombeo del agua de mar de cotas inferiores a cotas superiores de las salinas como se realizaba antaño en el proceso productivo del cultivo de la sal. Es notorio que, si no se realizan las labores de mantenimiento, protección y utilización de los mismos en los próximos años, consentiremos la pérdida para la sociedad actual y futura de unos elementos pertenecientes al patrimonio industrial tradicional y que, al mismo tiempo, forman parte de un valioso patrimonio cultural y paisajístico que definen las salinas en el archipiélago canario.

\section{Los aeromotores salineros}

El molino de viento denominado "aeromotor salinero" es una máquina eólica lenta que capta la energía cinética del viento para transformarla en energía mecánica para bombear el agua del mar procedente de un pozo o de un estancadero para luego elevarla posteriormente a los cocederos y a los tajos que se encuentran a una altura superior respecto al nivel de mar, donde luego cristaliza la sal por la evaporización del agua del mar debido a incidencia de la energía solar. En las salinas canarias coexisten varios tipos de molinos de viento y en cada tipo se distinguen varios elementos diferenciadores (Cabrera 2009)

\section{- Los edificios}

La morfología y las dimensiones del edificio que se conoce como base o pie, que asegura la estabilidad 
y el empotramiento de la torre del molino de viento aeromotor salinero canario son de dimensiones y de naturaleza variable. Se distinguen tres tipos de edificios con sus variantes respectivas:

- Edificio en Pozo: Es un volumen que surge como resultado de la prolongación del propio pozo, con forma cuadrada o circular y que sobresale en torno a 1.00 metro de altura respecto a la rasante del terreno.

- Edificio en Cubo: Este edificio supone una variante respecto al edificio descrito anteriormente y que adquiere su propia expresión arquitectónica al sobresalir en torno a los 2.50 metros a 3.00 metros de la rasante del terreno.

- Edificio en Mastaba: Es un volumen similar al edificio encubo, y sus paramentos verticales se construyen en talud para reforzar la estabilidad del edificio sin recurrir a los contrafuertes. Dicho edificio tiene un acceso hacia su interior que permite el registro y mantenimiento de la bomba de pistón.

\section{- Las torres}

Las torres de este tipo de molinos de viento son los elementos intermedios entre los rotores de aspas y las máquinas de bombeo, que habitualmente tienen forma piramidal triangulada construidas en estructura de madera y en escasas ocasiones de estructura metálica, cuya altura total oscila entre los 5.00 metros y los 10.00 metros, dependiendo fundamentalmente de la dimensión del diámetro del rotor de aspas. En este tipo de molinos de viento existen tres tipos de torres:

- Torre cuadrada de arriostre: Este tipo de torre es una estructura realizada en madera y es heredera directa de la torre cuadrada de madera del molino de viento harinero "La Molina". El esquema estructural de este tipo de torre está formado por cuatro columnas rectas de secciones rectangulares rigidizadas y trianguladas en su cara exterior por elementos continuos y lineales de madera. El arriostramiento de la torre se forma con piezas de secciones cuadradas o rectangulares transversales cuyas uniones se realizan en caja y en ocasiones se utilizan pernos y pasantes metálicos.

- Torre descuadrada de crucetas: Este tipo de torre es una estructura realizada en madera y es la más habitual en este tipo de molinos de viento salineros. El esquema estructural de este tipo de torre piramidal está compuesto por cuatro columnas rectas de secciones cuadradas, rectangulares o circulares que se disponen ligeramente inclinadas y arriostradas con piezas simples a modo de crucetas horizontales cuyas uniones se realizan en caja, en media madera o con pernos pasantes metálicos. El trazado geométrico habitual de la torre de madera en planta es básicamente de forma cuadrada. La construcción de las torres de madera es variada dependiendo de la combinación de las piezas de arriostramiento, encontrándose torres con dos o tres crucetas con arriostramiento en horizontal y con arriostramiento en vertical lateral, con crucetas horizontales y jabalcones o con crucetas horizontales y castillete. Este último modelo de torre de madera supone la máxima evolución de este tipo de torres de los molinos de viento salineros donde se origina una plataforma construida en madera que permite las labores de mantenimiento del rotor de aspas, del sistema de orientación, del sistema de regulación y del sistema de transmisión de este tipo de molinos de viento. asalineros.

- Torre metálica triangulada: Este tipo de torre tiene forma piramidal triangulada de hierro y supone una evolución estructural respecto a las torres de madera anteriormente descritas ya que por el cambio de material en su construcción permiten mayores alturas con menor sección de material y su empleo está estrechamente vinculado a la aparición en las islas Canarias del molino de viento denominado aeromotor "Tipo Americano". Su esquema estructural está compuesto por cuatro aristas laterales de secciones angulosas que se disponen ligeramente inclinadas y que se encuentran rigidizadas por elementos de pletinas o alambres de acero entrecruzados cuyas uniones se realizan con tornillos metálicos roscados. Este tipo de torres se caracterizan principalmente por su aspecto de ligereza debido fundamentalmente a las secciones de los perfiles angulares utilizados, presentado de esta forma menor incidencia de los vientos dominantes sobre los perfiles, pletinas y a veces cables empleados en la construcción de dichas torres, donde predominan por la elegancia y la ligereza frente a la robustez y la pesadez de los diversos tipos de las torres de madera empleadas en el resto de los molinos salineros. Sin embargo, en las salinas canarias se ha preferido mantener las torres de madera, para sustituir y adaptar los rotores de los antiguos molinos de viento salineros por los novedosos rotores multipala de chapa de los molinos de viento aeromotores "Tipo Americano"

\section{- Los rotores}

El rotor de aspas es el elemento más característico de un molino de viento salinero ya que constituye el sistema de captación de la energía eólica para transformarla en energía mecánica con el fin de bombear agua del mar hacia los cocederos y los tajos de las salinas. En este tipo de molinos de viento se han empleado varios tipos de rotores de aspas:

- Rotor de aspas de madera: Este tipo de rotor es heredero del molino de viento harinero "La Molina" de cuatro a seis aspas construidas en madera y con lonas. Este rotor es la solución más elemental y primitiva de los molinos de viento aeromotores salineros canarios. El esquema estructural se origina por el cruce de dos o tres palos largos denominados largueros y un conjunto de palos 
más pequeños Ilamados teleras o traviesas que son las que sostienen las lonas de tela de las aspas, que tienen una longitud aproximada de 3.00 metros, una anchura de 1.60 metros y los extremos de mismas se unen con cables entre sí. Para hacer frente a las variaciones de la velocidad del viento es necesario modificar la superficie de las lonas de las aspas, plegando o desplegando las lonas según la fuerza del viento.

- Rotor de aspas de velas: Este tipo de rotor es heredero del molino de viento mediterráneo de seis aspas construidas en madera y velas de lonas. Este rotor es la primera solución más habitual de los molinos de viento aeromotores salineros canarios. El esquema estructural se origina por el cruce de tres o cuatro palos largos denominados largueros que se arriostran con tirantes metálicos uniendo los extremos de los largueros de madera y que permiten arriostrar el rotor sin necesidad de utilizar vigas de madera de gran sección. Las velas de lonas tienen forma triangular y van sujetas a un lado por el lado más largo al larguero y por un vértice al larguero contiguo. Tanto las velas de lonas como los tirantes de sujeción de los largueros tienen cierta elasticidad para deformarse frente a la velocidad del viento lo que le confiere cierta cualidad de autorregulación frente a intensidades excesivas del viento. A veces las aspas de lonas triangulares se rompían con facilidad frente a velocidades excesivas del viento, aunque la velocidad de giro del rotor podía ser regulada por el procedimiento manual de soltar o recoger velas de las aspas del rotor. Estas operaciones de mantenimiento del rotor se realizan desde la cubierta del edificio o bien desde una plataforma instada en la torre de madera.

- Rotor multipala de chapa metálica: Este tipo de rotor es de fabricación artesanal y va ligado a la aparición del metal como elemento estructural y a la aparición comercial de los molinos de viento aeromotores "Tipo Americano". Este tipo molinos de viento de rotor multipala supuso una revolución respecto a las técnicas convencionales de los aeromotores destinados al bombeo de agua, ya que aumentaba la eficiencia de trabajo del mismo y permitía un mantenimiento más sencillo. Este rotor de aspa es la segunda solución más habitual de los molinos de viento "Salineros Canarios" que se caracteriza por su aspecto de ligereza y está formado por un número variable de aspas, que tiene un diámetro que oscila entre los 2.00 y 9.00 metros. Las aspas de este tipo de rotor son unas paletas metálicas de escasos milímetros de espesor y que tienen forma trapezoidal. Dichas aspas se agrupan habitualmente en grupos de tres, con un mínimo de dieciocho aspas y un máximo de veinticuatro y que presentan una forma cóncava hacia el viento. Dichas aspas se fijan mediante roblones metálicos sobre dos ó tres círculos metálicos, minimizando así las vibraciones del viento sobre las esbeltas aspas. Los círculos metálicos donde se fijan las aspas se sostienen sobre un mínimo de seis y un máximo de ocho brazos rígidos metálicos en forma triangular de celosía.

\section{- La maquinaria}

La maquinaria de este tipo de molinos de viento salineros destinados a bombear agua del mar hacia los cocederos y los tajos consta de una bomba metálica de aspiración de pistón que se pone en marcha por el movimiento lineal (de arriba hacia abajo) del vástago o vergajón metálico que proviene del sistema de transmisión. La bomba de aspiración se enlaza a una tubería metálica que se sitúa en el interior del pozo, tomadero o estancadero. Las bombas de aspiración son bombas de pistones y que están protegidas por un cilindro hueco metálico a modo de camisa, que disponen de un embolo y válvulas de cobre o de acero.

- Las proporciones geométricas de los aeromotores salineros.

Este tipo de molinos de viento guardan una proporción aproximada a la Ley de Tercios, que es una norma clásica de composición muy utilizada en el Renacimiento durante el siglo XV. Debido a la gran variedad y diversidad de tipos de los molinos de viento salineros que se construyen en las islas Canarias, existe variabilidad en cuanto a sus dimensiones (Cabrera 2009)

Gran parte de los molinos de viento salineros existentes en las salinas canarias responden principalmente a dos tipos: el molino de rotor de aspas de velas, que es el más utilizado y el molino de rotor aspas multipala de chapa metálica, ambos sobre una torre piramidal de madera.

- Molino de viento de rotor aspas de velas: La altura de las torres de este tipo de molinos de viento salineros está en función del diámetro del rotor de aspas empleado, con una altura de torre mínima que oscila entre los 6.00 metros a 8.00 metros, evitando las fluctuaciones y ondulaciones del viento sobre la superficie del terreno. En los molinos de viento aeromotores salineros canarios de rotor aspas de velas de lona y torre piramidal de madera la magnitud de referencia corresponde con el radio del rotor de aspas (B) siendo su dimensión máxima de 3.00 metros. La altura total de este tipo de molinos de viento salineros es de aproximadamente dos diámetros la dimensión del rotor de aspas, estando desfasados en vertical la dimensión de un tercio de la dimensión del radio del rotor de aspas. [figura 4 y figura 5]

- Molino de viento de rotor multipala: Este tipo de aeromotores salineros canarios de rotor multipala de chapa metálica y torre piramidal de madera la magnitud de referencia corresponde con el radio del rotor de aspas (B) siendo su dimensión máxima de 3.00 metros. La altura total de este tipo de molinos de viento es de aproximadamente dos diámetros la dimensión del rotor de aspas. Con respecto a las proporciones geométricas, este tipo de molino de viento salinero se organiza en dos partes bien diferenciadas que son el rotor de aspas y la torre piramidal de madera. Las proporciones geométricas que se muestran a continuación dependen de las tolerancias existentes en la construcción de este tipo de molinos de viento. [figura 6 y figura 7] 


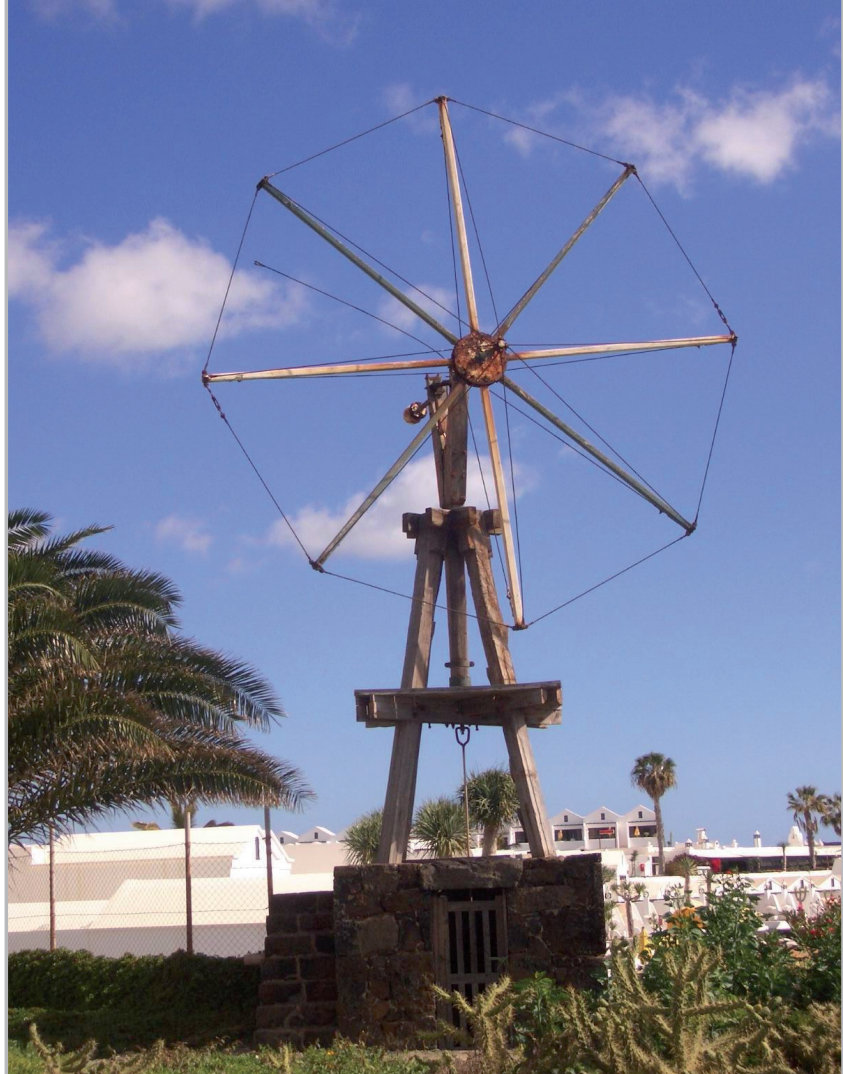

Figura 4.- Aaeromotor salinero canario de rotor de aspas de velas. Costa Teguise. Lanzarote Fotografía: Víctor M, Cabrera García

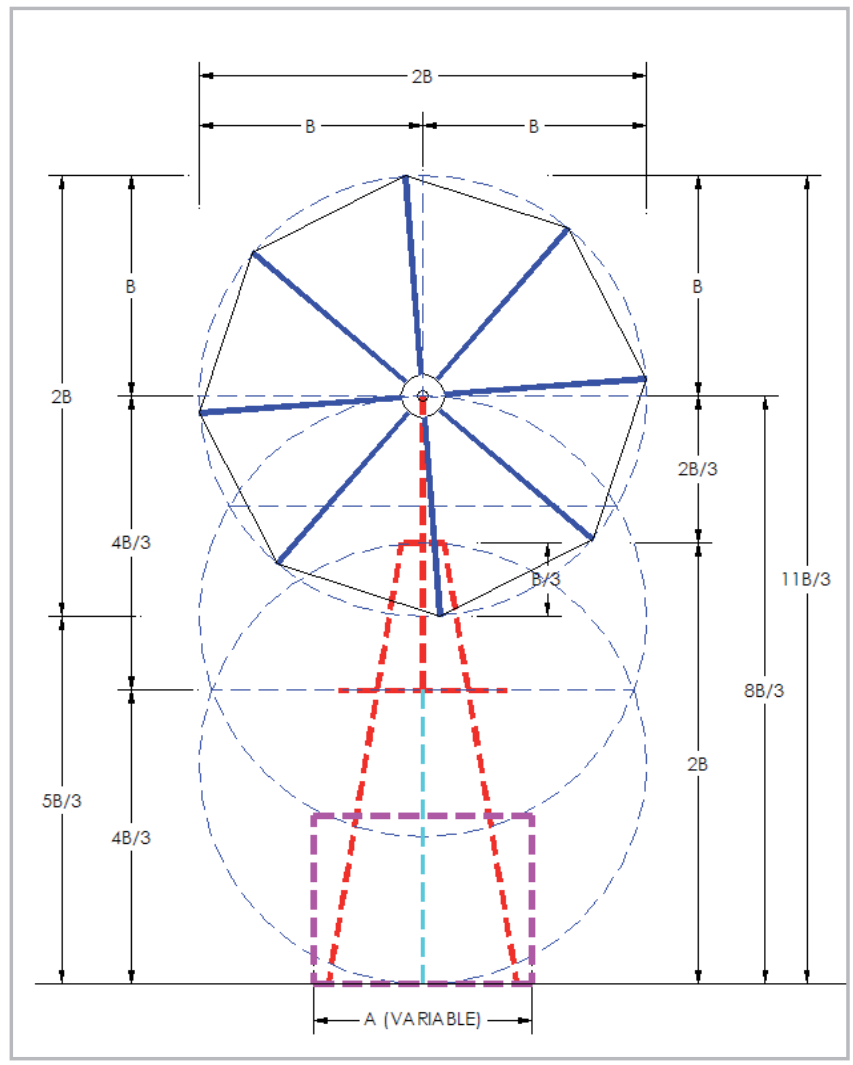

Figura 5.- Proporciones geométricas del aeromotor salinero canario de rotor de aspas de velas. Dibujo: Víctor M. Cabrera García

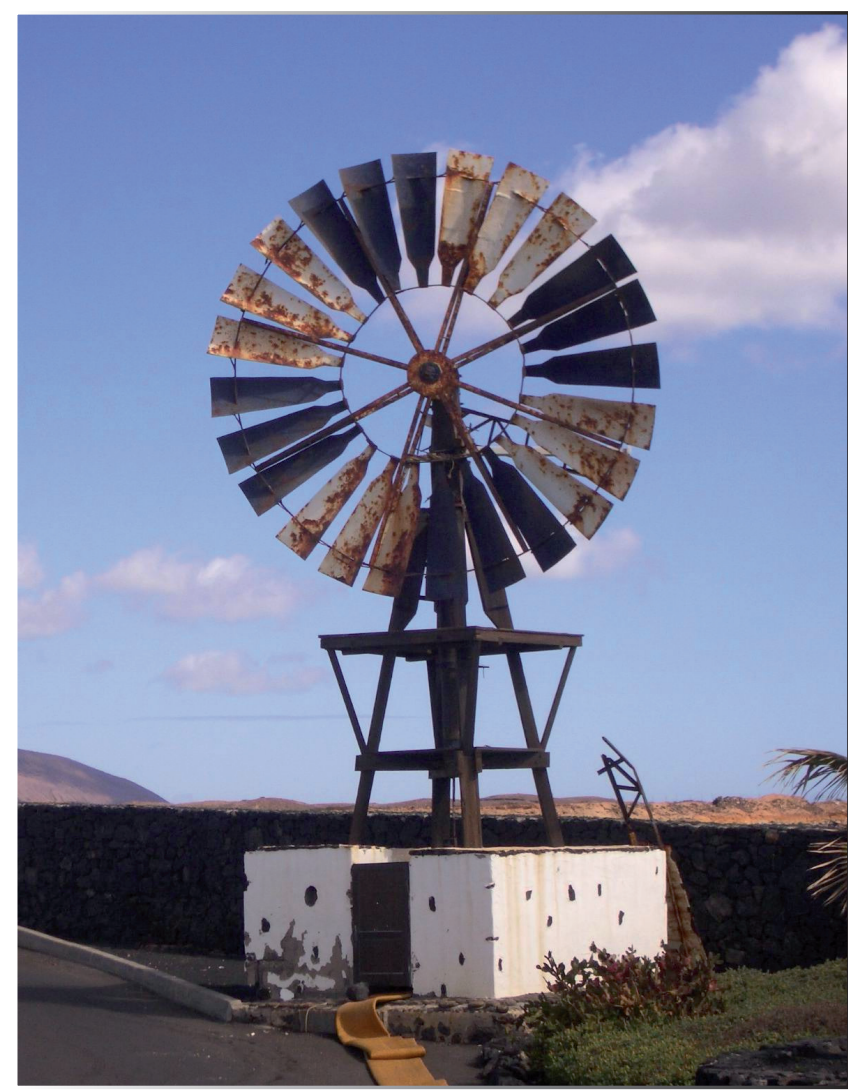

Figura 6.- Aaeromotor salinero canario de rotor de aspas multipalas metálicas. Costa Teguise. Lanzarote.Fotografía: Víctor M. Cabrera García

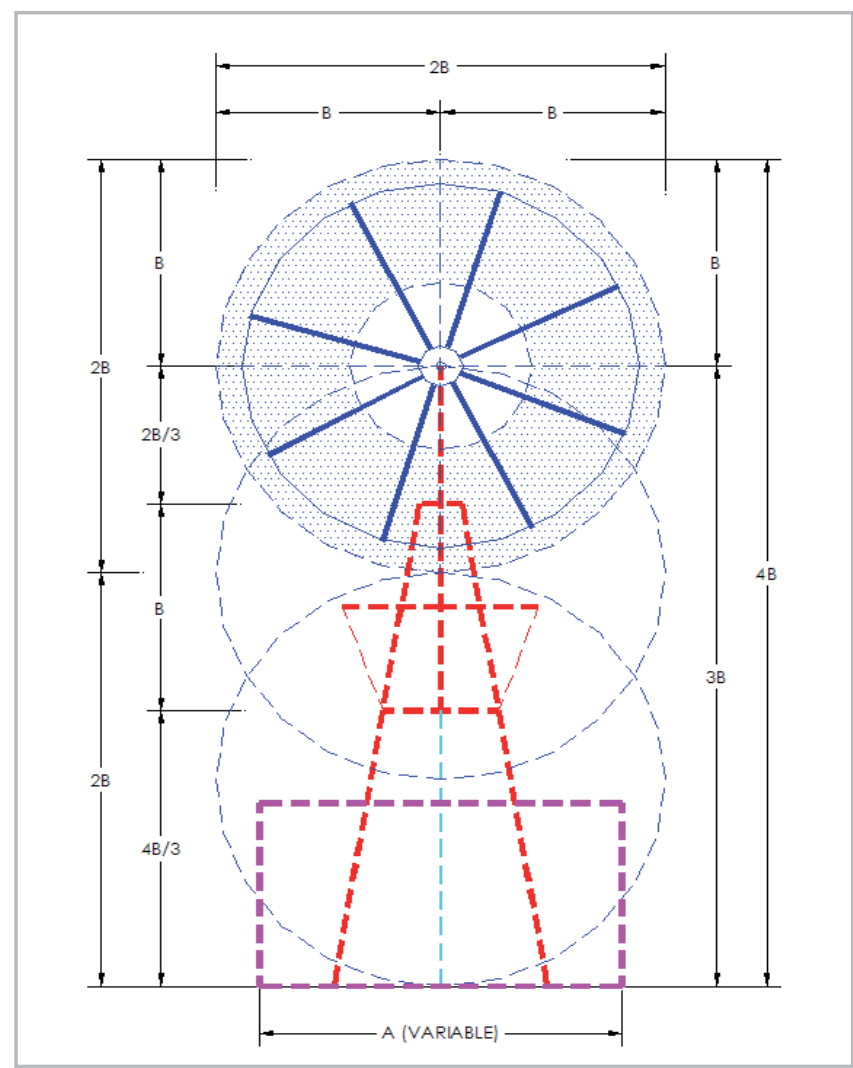

Figura 7.- Proporciones geométricas del aeromotor salinero canario de rotor de aspas mulitipala de chapa metálica. Dibujo: Víctor M. Cabrera García 


\section{Resumen del estado de la cuestión}

Los molinos de viento tradicionales, tanto los harineros como los salineros son unas construcciones singulares de la arquitectura popular canaria que poseen ciertos valores históricos, arquitectónicos, etnográficos y son testigos singulares de la evolución de la cultura canaria. Estos elementos que también pertenecen al patrimonio industrial de las islas pertenecen al pasado ya que han desaparecido los modos de vida a los que iban ligados y no son útiles para la sociedad actual. La falta de estudio e inventario de los mismos, la falta de uso, así como la fragilidad relacionada con el envejecimiento de todos los materiales empleados en su construcción los hacen especialmente vulnerables. Los molinos de viento tradicionales que se construyeron en las islas Canarias son unas construcciones que sirven de testigo de nuestra cultura tradicional popular, por esta razón los molinos de viento que han sobrevivido al paso del tiempo nos ofrecen numerosos datos sobre la economía y las técnicas artesanales locales que se utilizaban en épocas pasadas en el Archipiélago Canario, por lo tanto estas construcciones singulares son un legado que hemos recibido de nuestros antecesores, nos pertenece a todos y forman parte de nuestra historia e identidad como pueblo, por lo que debemos de realizar entre todos (los ciudadanos y las administraciones públicas) un gran esfuerzo para conocerlos, protegerlos, conservarlos y difundirlos.

Las funciones para las que se construyeron los antiguos molinos están prácticamente extinguidas y la rentabilidad de la actividad productiva para la que fueron creados permitió su mantenimiento durante un determinado periodo de tiempo, hasta que las nuevas y más potentes tecnologías desplazaran la producción de las antiguas del mercado, y obligaran a estas últimas a caer en desuso. Son numerosas las iniciativas desarrolladas hasta la fecha que han alcanzado el objetivo de recuperar el aspecto original de las edificaciones tras su catalogación como elemento patrimonial. Pero, en demasiadas ocasiones, el proyecto se limita a dar únicamente ese primer paso: la restauración del edificio. Sin una fuente permanente de ingresos procedente de una actividad asociada al elemento restaurado, que permita el mantenimiento de esas instalaciones rehabilitadas, el patrimonio recuperado está destinado a deteriorarse una vez más $y$, muy probablemente, a desaparecer con el paso del tiempo (Lalanda; Gomez-Elvira; De Los Rios 2010)

Según la Ley 4/1999 sobre el Patrimonio Histórico de Canarias que posteriormente es modificada por el Ley 11/2002 del 21 de noviembre cita lo siguiente:

"Se declaran bienes de interés cultural del patrimonio histórico canario aquellos bienes que ostenten notorios valores históricos, arquitectónicos, artísticos, arqueológicos, etnográficos o paleontológicos o que constituyan testimonios singulares de la cultura canaria".
Las salinas canarias son espacios singulares que reúnen los valores anteriormente citados y es por ello que se han tramitado varios expedientes de incoación como Bienes de Interés Cultural en determinadas categorías.

-Isla de Lanzarote:

Salinas de Ozorla, en TM de Haría, fecha de incoación: 09/03/2004

Salinas de Naos, en TM de Arrecife, fecha de incoación: 17/06/2003

Salinas de Janubio, TM de Yaiza, fecha de incoación: $12 / 06 / 2003$

Salinas de la Bufona, TM de Arrecife, fecha de incoación: 17/06/2003

Salinas del Río, TM de Haría, fecha de incoación: $03 / 12 / 2002$

Salinas de las Caletas, TM de Teguise, fecha de incoación: 29/10/2002

Salinas del Tío Joaquín, salinas de don Pancho, TM de Teguise, fecha de incoación: 29/10/2002

Salinas de los Agujeros, TM de Teguise, fecha de incoación: 29/10/2002

Salinas de el Río o salinas Bajo el Risco, TM de Haría, fecha de incoación: 11/06/2003

Zona paleontológica de las salinas del Janubio, TM de Yaiza, fecha de incoación: 19/06/2003

\section{- Isla de Gran Canaria}

Salinas del Bufadero, TM de Arucas, fecha de incoación: 03/12/1997

Salinas de Tenefé, TM de Santa Lucía de Tirajana, fecha de incoación: 03/12/1997

Salinas de Arinaga, TM de Agüimes, fecha de incoación: 15/06/2001

-Isla de Fuerteventura

Salinas del Carmen, TM de La Antigua, fecha de incoación: 14/08/2002

De todos lo expedientes incoados tan solo las salinas de Arigana en el municipio de Agüimes en la isla de Gran Canaria ha sido declarada como BIC en la categoría de "Sitio Etnográfico" según el Decreto 131/2008 del 10 de junio. El resto de los expedientes siguen su tramitación.

Debido a los complejos trámites en las resoluciones de los expedientes respecto a protección de estos espacios singulares como Bien de Interés Cultural que se alargan en el tiempo y la manifiesta erosión de los diversos elementos de las salinas que se están produciendo ante el abandono de las mismas por falta de mantenimiento $i$ Existen alternativas distintas a las ya ensayadas para recuperar, incorporar, reutilizar las salinas canarias y en especial, a los molinos de viento tradicionales denominados aeromotores salineros que mayoritariamente se encuentran actualmente abandonados y en estado de ruinas para la actual sociedad canaria? 


\section{Propuestas para su revitalización}

\section{-Revitalizar las salinas a través de la gastronomía}

Actualmente en la gastronomía de gourmet se está empezando a utilizar la denominada "espuma de sal" o "flor de sal", que es un tipo de sal más esponjosa que la sal convencional de grano. La flor de sal o sal en escamas se diferencia de otras sales por su forma y estructura plana y no por su composición. Se obtiene mediante la evaporación del agua de una solución salada en la superficie de los tajos. Una vez que aumenta la salinidad del agua del mar en los tajos debido fundamentalmente a la evaporización, se recogen los cristales de la capa superficial que tienen una forma de escama y que pueden alcanzar unos pocos milímetros de tamaño. Estas escamas son muy quebradizas e introducidas en la boca no sólo aportarán sabor a sal, sino que además serán agradablemente crujientes. Y es que esta última característica es la que realmente diferencia a la sal en escamas o a la flor de sal de las demás sales: su capacidad de aportar una textura crujiente a un bocado en la degustación de los platos. Estas escamas de sal son además muy decorativas, algo que hace que sean un añadido ideal para la presentación de los platos refinados.

La sal en escamas suele tener un precio relativamente elevado dado que es costoso producirla en grandes cantidades ya que su recogida se realiza a mano de los maestros salineros. Su sabor no tiene por qué ser diferente al de cualquier otra sal y por ello no se suele usar para cocinar. Una vez disuelta en agua, no es diferente de otra sal cualquiera y por ello es más lógico usar para cocinar sales más económicas y reservar la sal en escamas para ser espolvoreada en pequeñas cantidades sobre los platos refinados respecto a su presentación a los comensales. Actualmente se está produciendo una revolución en cuanto a la elección y el empleo de diferentes tipos de sal, presentadas y comercializadas al natural, con carbón, con tomate, ahumada, con jengibre, de distintos colores, etc.

\section{— Revitalizar los molinos de viento aeromotores salineros}

Un molino de viento es una máquina que convierte una determinada forma de energía procedente de su fuerza motora, el viento, el agua, un combustible, etc., en energía mecánica, capaz de mover un mecanismo que produce un trabajo útil para el hombre. Aunque el término molino está relacionado con "moler", se ha aplicado esta denominación a toda máquina cuya energía se capta con un dispositivo giratorio, aunque su objetivo último no sea moler grano (Valera 2010). El mejor método para la conservación de los edificios procedentes de la cultura tradicional es mantenerlos con vida. Los aeromotores salineros tradicionales pertenecen al pasado, fundamentalmente porque su uso no se corresponde con las necesidades de la vida actual. En la actualidad, los molinos de viento se utilizan fundamentalmente para generar energía eléctrica a través de los aerogeneradores de pequeñas, medias y grandes potencias.

Como alternativa a las diversas estrategias existentes destinadas a procurar la conservación de estos elementos de la arquitectura popular se propone una iniciativa que consiste en recuperar el funcionamiento de estos tradicionales molinos de viento dotándoles de un nuevo uso, es decir, implantándoles una tecnología que les permita producir energía eléctrica mediante el acoplamiento de un generador de baja potencia, iniciativa similar a la propuesta por el Ayuntamiento de Campos en Palma de Mallorca con el Proyecto, Dirección de Obra y GestiónExplotación del Proyecto de Recuperación Patrimonial "Molins de Campos" en Mallorca, realizado por la Ingeniería y Consultoría Técnica Pascual Esteva SLL en el año 2000. El proyecto descrito contemplaba la rehabilitación estructural de diversas máquinas eólicas, además de concederle una prioridad ambiental integral ya que genera energía eléctrica a través de la energía cinética del viento, limpia y renovable, al tiempo que se mejora el entorno con una serie de actuaciones colaterales, impulsando el empleo y que fue fruto de la experiencia adquirida en la ejecución de un estudio experimental desarrollado como consecuencia del convenio de colaboración entre el Ministerio de Medio Ambiente, el Instituto para la Diversificación y Ahorro de la Energía, el Ayuntamiento de Campos y la empresa privada GESA-Endesa (Cabrera 2016).

De esta forma se consigue dar respuesta a la inoperatividad actualdelostradicionalesmolinos deviento,incorporándoles un nuevo uso y que es compatible con las necesidades sociales actuales en el interés por la obtención de energía eléctrica a través de las energías renovables y es aquí donde los actuales aerogeneradores aportan su tecnología a los molinos de viento tradicionales permitiéndoles recuperar su funcionamiento. La energía eléctrica producida se podría utilizar para dar suministro energético a la iluminación artificial a disponer en las salinas, creando nuevos escenarios paisajísticos de contemplación nocturna de las mismas como complemento a las actividades diurnas que se realizan en las salinas.

\section{— Revitalizar las salinas través del paisajismo}

Existe una experiencia singular de un paisaje híbrido de naturaleza y cultura creado según las directrices de un proyecto denominado "La Tancada" en los campos de sal del Parque Natural del Ebro en Tarragona. El Proyecto se sitúa en el extremo sur del Río Ebro Delta, uno de los ríos más importantes y caudalosos de la Península Ibérica. Delta es un paso crucial en la ruta de migración de aves de Europa Occidental. Bajo la necesidad de restaurar un área natural, el proyecto tiene como objetivo transformar las piscinas de una antigua fábrica de pescado en un paisaje liquido, que intenta preservar las poblaciones existentes, proteger la flora y multiplicar las condiciones de hábitat para diversas aves acuáticas, creando pequeños lagos de mar. Se trata 
de una construcción de un paisaje como un ecosistema artificial, que propone formas coreográficas para ser recorridas, dentro de una narrativa que invita a explorar las lagunas intermareales, campos de sales y cristalizaciones, flamencos, etc.

En el año 2009 se produjo un acuerdo entre la Fundación Natural Catalunya Caixa, el Ministerio de Medio Ambiente de España, el Parque Natural Delte de l'Ebre y el Instituto de investigación Agrícola lograron que junto con la UE se creara el denominado programa LIFE con la finalidad de restaurar este sitio y otras áreas naturales cercanas. Este proyecto tuvo como objetivo proporcionar hábitat a diversas especies protegidas de la flora y de la fauna que están seriamente amenazadas a desaparecer. Los científicos a cargo de la misión solicitaron la necesidad de diversificar el binomio tierra-agua con el fin de multiplicar las condiciones del hábitat. Así, redistribuyendo la arena en los diques, el proyecto implementa cuatro distintos tipos de secciones transversales del terreno con diversas profundidades en las lagunas, regímenes de inundaciones y laderas.

Del proyecto LIFE surge otro proyecto denominado "MON NATURA" que consistió en crear un Centro de Interpretación para convertir la explanada existente extremadamente plana expuesta en un paisaje líquido, construida como un archipiélago de cuatro islas que contienen y secuencian la propuesta interpretativa del programa. Se creó un paisaje consubstancial líquido y salado que realiza una magnífica interacción con el ciclo de luz. El diseño del proyecto construye un híbrido de naturaleza y cultura que dignifican las peculiaridades del sitio, tanto en términos de los ecosistemas artificiales para las especies en peligro de extinción como en la construcción de este paisaje naturalartificial que propone formas alternativas y sugerentes en el sitio para los visitantes, dentro de un contexto que los invita a explorar este peculiar paisaje: lagunas intermareales, campos de sales y cristalizaciones, así como la presencia de flamencos, charranes, etc.

\section{- Revitalizar a través del turismo cultural}

Según la fundación COTEC para la innovación tecnológica, en su informe del año 2010, la innovación en el ámbito del patrimonio, un sector tradicionalmente más reactivo que proactivo y acostumbrado a las políticas de subvención a fondo perdido, no es una opción, sino una acuciante necesidad en una doble dirección: innovación para hacer económicamente viable la conservación de tanto patrimonio, desarrollando nuevos modelos de explotación y gestión sostenible de recursos patrimoniales; en innovación para mejorar y ampliar la demanda de conocimiento y de turismo cultural a través de una oferta planificada y de calidad, capaz de convertir los recursos patrimoniales en productos culturales y económicos, y capaz también de adaptar los comportamientos de la demanda a las necesidades de preservación de los valores culturales del patrimonio. Conservar un espacio con valor patrimonial por su paisaje natural no supone prohibir el desarrollo de nuevas actividades, ya que recuperar y proteger sin usos resulta ser una acción en vano.

Las salinas y su entorno, frecuentemente enclavadas en espacios marismeños, constituyen uno de los hábitats más singulares del planeta. Un lugar donde las condiciones físicas son excepcionales y donde la actividad humana tradicional ha conseguido un modelo de intervención que, lejos de degradar el ambiente, lo mejora en algunos aspectos. Pero desgraciadamente, estos enclaves de carácter singular, donde confluyen intereses económicos, sociales, ecológicos y culturales, se encuentran seriamente amenazados. La amenaza viene de la mano de la presión urbanística y de la decadencia de la industria salinera tradicional que, hasta el momento, había logrado poner límites a la ocupación salvaje del litoral. La pérdida del manejo salinero, garantizando los flujos y controlando las láminas de agua, despoja a estas zonas del motor de su subsistencia. La compleja y antigua maquinaria que hacía funcionar estos magníficos paisajes de agua se abandona paulatinamente, arrastrando consigo el paisaje de estas marismas artificiales. La declaración de gran parte de estos parajes como espacios naturales debería, pues, completarse con un conjunto de medidas que garanticen al menos el mantenimiento de la forma y la funcionalidad de esta singular arquitectura de la zona húmeda. Las salinas pueden situarse sobre una gran diversidad de hábitats costeros, desde las costas rocosas hasta lagunas, albuferas y marismas de agua salobre, salina o hipersalina. La mayor parte de las salinas históricas se desarrollaron sobre espacios hoy denominados como "zonas húmedas", superficies de terreno cubiertas temporal o permanentemente por el agua que, antaño, eran zonas denostadas y que, en la actualidad, se han convertido en reductos de indudable valor ecológico. Sin embargo, una parte importante de las salinas canarias se instalaron sobre costas rocosas, donde no existía ni el menor asomo de zonas húmedas. En tales casos, la construcción de las salinas no solo permite el mantenimiento de las especies vegetales y animales que había anteriormente, sino que aumenta la diversidad del hábitat con sus superficies de aguas someras, y con ello la riqueza y variedad de vegetales y animales (Marín; Luengo 1994)

La calidad del recurso (o nivel de conservación) influirá directamente en la calidad de la experiencia que viva quien visite los monumentos (que puede ser tanto población local como externa). En base a la satisfacción que pueda sentir el visitante, éste regresará o no para repetir la experiencia, o podrá o no recomendar a su amigo la visita. El incremento del número de visitas tiene, en principio, un evidente impacto económico positivo, ya sea en los ingresos que se redirijan de manera directa al mantenimiento del recurso, o bien, en las cajas de los restaurantes, hoteles y demás actividades que se desarrollen en las cercanías del patrimonio en cuestión, cuyos ingresos deberán entenderse como producto indirecto de la revalorización y dinamización del patrimonio. El beneficio económico puede plantearse así de manera directa o indirecta y, en 
cualquier caso, dependiente de la calidad del recurso y de la experiencia del visitante. En relación con el incremento de visitas, es importante también tener en cuenta la posibilidad de generar un impacto negativo si se supera la capacidad de carga del recurso, la cual dependerá, en parte, de la manera en que se haya acondicionado el lugar para soportar la afluencia de personas y de la forma en que los visitantes conozcan y pongan en práctica una conducta favorable a la conservación (Lalanda; Gomez-Elvira; De Los Rios 2010)

\section{Conclusión}

El patrimonio intangible asociado a las salinas configura también un bien cultural de primer orden, cuya continuidad depende de que la actividad y su cultura material (patrimonio inmueble) se mantengan en activo. Por tanto, la conservación de las salinas supone también una garantía de continuidad para un oficio, el de los maestros salineros, que guarda una importante carga histórica ligada a los inicios de la actividad, los conocimientos acumulados y transmitidos durante varias generaciones por parte de los maestros salineros canarios como parte inseparable de este legado histórico. Las salinas representan también un modelo de interacción ambiental entre el aprovechamiento de los recursos y las condiciones del medio natural. De esta manera se comportan como marisma artificial, generando un ecosistema singular en el que tienen su hábitat un número importantes de especies naturales y vegetales, que incorporan valores añadidos a los reseñados, especialmente con la fauna, como estación de paso y avituallamiento de las aves migratorias.

Las estrategias clásicas de conservación de las salinas suelen ser de dos tipos, por un lado, se apuesta por mantener la actividad en las salinas, cada vez más en declive, aunque no se rijan por la productividad de las mismas y, por otro lado, mediante la protección por la legislación según indica la ley de Patrimonio Histórico de Canarias. Sin embargo, se ha constatado que, aunque se haya incoado numerosos expedientes para proteger las salinas, existen varios expedientes que han caducado, por lo que se encuentran en la actualidad en un vacío jurídico importante, lo que favorece la erosión de los diferentes elementos que componen estos espacios singulares al carecer de actividad y de mantenimiento de las instalaciones. Cabe decir que, de los expedientes tramitados, tan solo se ha declarado como BIC en la categoría de "Sitio Etnográfico" la salina de Arinaga, en el municipio de Agüimes, en la isla de Gran Canaria.

Ante el manifiesto estado ruinoso que presentan las salinas canarias debido al abandono por parte de la sociedad actual de las mismas debido fundamentalmente a falta de actividad, se proponen alternativas que difieren de las estrategias clásicas de conservación. La revitalización de las salinas mediante la gastronomía de gourmet utilizando la "espuma de sal" o "flor de sal", que es un tipo de sal más esponjosa que la sal convencional de grano, aporta una textura crujiente a los platos además de ser un recurso decorativo en la presentación de los mismos. Actualmente se está produciendo una revolución gastronómica en cuanto a la elección y empleo de diferentes tipos de sal de la mano de cocineros de referencia y este tipo de acciones pueden ayudar a reactivar la actividad en las salinas.

Revitalizar las salinas canarias mediante el paisajismo es otra de las alternativas que se proponen con la intención de incentivar otro sector como es el turismo cultural, para que estos espacios salineros puedan convertirse en un reclamo tanto para los lugareños como para los nuevos visitantes. Se propone recuperar entre otros, el funcionamiento de los tradicionales molinos de viento salineros que actualmente están abandonados debido a la falta de actividad, dotándoles de un nuevo uso, es decir, implantándoles una tecnología que les permita producir energía eléctrica, y que al mismo tiempo es compatible con las necesidades sociales actuales en el interés por la obtención de energía eléctrica a través de las energías renovables. La propuesta de crear nuevos paisajes ajardinados de carácter líquido a partir de los ya existentes e iluminados artificialmente en horario nocturno podría convertirse en nuevos polos de atracción para el turismo cultural creando nuevas experiencias tanto diurnas como nocturnas en estos singulares espacios marítimo-terrestres.

La energía eléctrica necesaria para permitir iluminar artificialmente las salinas en horario nocturno facilitaría la creación de nuevas rutas peatonales empleando diferentes tonalidades de colores en los tajos y los cocederos creando recorridos cromáticos de luz artificial que tendría que obtenerse a partir de las energías renovables, de una parte, de la energía eólica obtenida a través de los aeromotores salineros reconvertidos a aerogeneradores de baja potencia y de otra parte, de la energía fotovoltaica. El empleo de estas dos energías renovables y limpias permitirá obtener la energía necesaria para poder iluminar artificialmente mediante lámparas Led (bajo consumo energético) a estos espacios etnográficos tan singulares, generando nuevos escenarios paisajísticos de contemplación nocturna de las salinas. Esta propuesta posibilita dar respuesta a la inoperatividad actual de los diversos tipos aeromotores salineros tradicionales, incorporándoles un nuevo uso y creando un nuevo reclamo turístico en la contemplación de unos paisajes marinos tan singulares y domesticados por la humanidad en la obtención de la sal.

Las diferentes propuestas de revitalización indicadas posibilitarán dar respuesta a la inoperatividad actual que tienen los diferentes elementos constructivos que conforman las salinas canarias, fomentando recuperar lo que aún no se ha perdido de estos espacios tan singulares pertenecientes al patrimonio industrial tradicional canario, permitiendo alargarles su vida útil y así poder legarlos con garantías de conservación para las generaciones futuras.

"Lo que sabemos es una gota de agua, lo que ignoramos es el océano"

ISAAC NEWTON 


\section{Bibliografía}

Arquitectura y Paisaje: Campos de sal "La Tancada". http://www. archdaily.co/co/02-365898/arquitectura-y-paisaje-camposde-sal-la-tancada-convertidos-en-un-paisaje-liquido-por-emf [Consulta 20.11.2018].

BOLETÍN OFICICIAL DE CANARIAS: DECRETO 131/2008, de 10 de junio, por el que se declara Bien de Interés Cultural, con categoría de Sitio Etnológico "Las Salinas de Arinaga", situadas en el término municipal de Agüimes, isla de Gran Canaria, delimitando su entorno de protección.

CABRERA GARCÍA, V. M. (2009) La Arquitectura del Viento en Canarias. Los molinos de viento. Clasificación, funcionalidad y aspectos constructivos. Tesis Doctoral, Universidad de Las Palmas de Gran Canaria.

CABRERA GARCÍA, V. M. (2010). Molinos de viento en las Islas Canarias. Tenerife. Editorial: Ediciones Idea S.A. Colección: Territorio Canario.

CABRERA GARCÍA, V. M. (2016). Aprovechamiento energético de los molinos de viento tradicionales de las Islas Canarias. Editorial: Cogiti, Revista Técnica Industrial, № 317, pp 58-66.

CENTRO DE EDUCACIÓN DE PERSONAS ADULTAS DE AGÜIMES INGENIO. http://www3.gobiernodecanarias.org/medusa/ ecoblog/ssuaseg/files/2018/03/unidad-didactica-las-salinascanarias.pdf. [Consulta 05.10.2018]

CONVENIO EUROPEO del Paisaje, Florencia 2000.

https://rm.coe.int/CoERMPublicCommonSearchServices/Disp layDCTMContent?documentld $=09000016802 \mathrm{f3fbd}$ [Consulta 15.11.2018]

ESPAÑA. LEY 4/1999, de 15 de marzo, de PATRIMONIO HISTÓRICO DE CANARIAS, modificada por La LEY 11/2002, de 21 de noviembre. Gobierno de Canarias.

Fundación COTEC para la innovación. https://www.madrimasd. org/empleo/documentos/doc/informe COTEC 2010.pdf [Consulta 31.10.2018]

https://www.eldiario.es/viajarahora/DESTINO-CANARIASGran-Canaria-salinas-todas-Bocacangrejo-Playa-FloridaTenefe-Arinaga-tajos-cocederos-molinos-Isleta-Las-PalmasConfital-Burrero-Arucas-Aguimes-Santa-Lucia-PozoIzquierdo 0 471403515.html [Consulta 02.10.2018]

https://www.cocinista.es/web/es/enciclopedia-cocinista/ ingredientes-del-mundo/flor-de-sal.html [Consulta 16.11.2018]

LALANDA ORDÓÑEZ, R, GÓMEZ-ELVIRA GONZÁLEZ, M.A, DE LOS RIOS CARMENADO, I (2010). Instrumento de apoyo para la puesta en valor y gestión sostenible de antiguos edificios de valor histórico-cultural en España: Marco ordenador, objetivos e indicadores. Comunicación. XIV International Congress on project engineering. Madrid.
Las sales groumet: https://www.salriera.es/sales-gourmet/ [Consulta 20.11.2018]

MARÍN, C, LUENGO, A. (1994) "El jardín de la sal". Santa Cruz de Tenerife. Editorial: Ecotopía.

ROMÁN LÓPEZ, E." 1994). “El patrimonio territorial como base para un nuevo modelo de desarrollo territorial. Las salinas en el territorio: paisaje y patrimonio". VII Congreso Internacional de Ordenación del Territorio, Madrid 2014.

PASCUAL TORTELLÁ, J. (2003). “Reconversión de molinos de viento tradicionales de extracción de agua de Campos, Mallorca, para la producción de energía eléctrica". IV Congreso Internacional de Molinología. Volumen: 2, 2005, 369-380.

VALERA MARTÍNEZ-SANTOS, F. (2010). Principios físicos y tecnología del molino de viento. https://www.campodecriptana.info/.../Fisicay-tecnologia-del-molino-de-viento.pdf. [Consulta 17.01.2017]

\section{Autor}

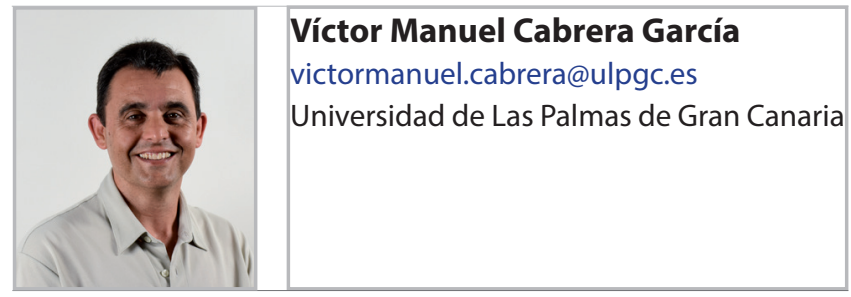

Doctor Arquitecto en Restauración y Rehabilitación Arquitectónica por la Universidad de Las Palmas de Gran Canaria. Arquitecto con las especialidades de Edificación y Urbanismo por la Universidad de Las Palmas de Gran Canaria. Técnico Superior en Sistemas Electrotécnicos y Automatizados por el I.E.S Felo Monzón en Las Palmas de Gran Canaria. Arquitecto especialista en diseño, cálculo y ejecución de estructuras en la edificación. Ha sido Profesor de la Escuela de Arquitectura de la Universidad Europea de Canarias y actualmente es Profesor Ayudante Doctor del Departamento de Construcción Arquitectónica de la Escuela de Arquitectura de la Universidad de Las Palmas de Gran Canaria. Investigador del Grupo de Investigación Reconocido URCAPES de la Universidad de Las Palmas de Gran Canaria, Investigador del Grupo de Investigación "Arquitectura y Desarrollo Turismo Sostenible" de la Universidad Europea de Canarias e Investigador de la Asociación para la Conservación y Estudio de los Molinos "ACEM", entidad sin ánimo de lucro interesada en el estudio de los molinos y su entorno, los edificios, los elementos y los mecanismos que utilicen para su funcionamiento las distintas energías tradicionales. 